\title{
Nachsorgeintervall auf drei Jahre verkürzen?
}

Hintergrund: Die Screeningkoloskopie und die konsequente Adenomabtragung mit konsekutiver -nachsorge hat zu einer Reduktion des kolorektalen Karzinoms (KRK) und der Sterblichkeit des KRK geführt. Die verschiedenen nationalen Leitlinien schreiben unterschiedliche Nachsorgeintervalle vor. Viele Kollegen weichen aber gerade bei kleinen oder weniger als zwei Adenomen von diesen Leitlinien ab und untersuchen früher nach.

Patienten und Methoden: Dieser „Nachsorge“-Problematik ist die hier vorgestellte Studie nachgegangen. Die Autoren haben die Häufigkeit und die Ergebnisse früherer koloskopischer Nachsorge kleiner Low-Risk-Polypen im Zusammenhang mit einer großen Chemopräventionsstudie mit einer Gesamtzahl von 1.560 Patienten überprüft.

Ergebnisse: $38 \%$ der in die Studie eingeschlossenen Patienten mit einem Low-Risk-Adenom wurden schon nach drei Jahren nachkontrolliert, anstatt nach den vorgesehenen fünf Jahren. In dieser früh untersuchten Gruppe fand sich kein statistisch signifikanter Unterschied,

Anderson JC, Baron JA, Ahnen DJ et al. Factors Associated With Shorter Colonoscopy Surveillance Intervals for Patients With Low-Risk Colorectal Adenomas and Effects on Outcome. Gastroenterology 2017; 152: 1933-43 was die Häufigkeit von fortgeschrittenen Adenomen ( $38 \%$ vs. $41 \%$ ) betrifft, obwohl in der Kurzintervallgruppe statistisch signifikant mehr Menschen mit familiärem Risiko für eine KRK gefunden wurden ( $\triangleright$ Abb.1).

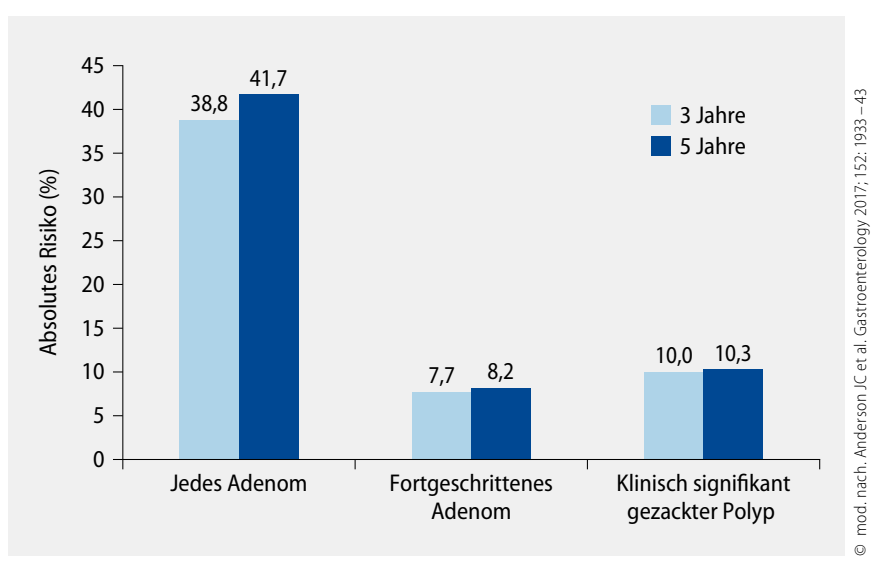

1 Ergebnisse der Nachkontrollen nach drei und fünf Jahren.

Schlussfolgerungen: Die Autoren spekulieren über die Gründe der früheren Koloskopie, die man anhand der Daten erfassen kann: Patienten mit einer familiären Belastung, mit mehr als einem Adenom, und solche, deren Adenome $>5 \mathrm{~mm}$ waren, wurden ebenso früher untersucht wie Afrikaner und Asiaten. Prozedurenabhängige Faktoren, die eine frühere Untersuchung triggerten, waren vor allem die unzureichende Darmvorbereitung, wobei die Zahl der schlecht vorbereiteten Patienten sehr niedrig war aufgrund des Studienprotokolls. Nichtgastroenterologen haben ebenfalls häufiger früher untersucht als Gastroenterologen.

\section{- Kommentar von Dieter Schilling, Mannheim}

\section{Frühere Untersuchung bringt bei Low-Risk keinen Vorteil}

Die Kernbotschaft der Arbeit ist, dass eine frühere Untersuchung in einer Low-Risk-Gruppe keinen Vorteil für den Patienten bringt. Somit sensibilisiert die Studie für ein wichtiges Thema in Bezug auf die Vorsorgekoloskopie - die Überkontrolle der Menschen, die kein hohes Risiko haben. Eine Methode wie die Vorsorgekoloskopie, die auf dem besten Weg ist, auch ein evidenzbasiertes Niveau zu erreichen, darf nicht überstrapaziert werden und letztlich durch mögliche Komplikationen in Misskredit geraten. Ein Editorial von David Lieberman zu dieser Arbeit in Gastroenterology bringt es mit der Head Line: „Low Risk is real low risk " auf den Punkt . In USA kennt man Zahlen, dass der "overuse" der Kontrollkoloskopie bei $25 \%$ aller Koloskopien liegt. Dies ist neben der unnötigen Belastung der Patienten (Probanden?) auch ein erheblicher finanzieller Aufwand, den in Deutschland die Solidargemeinschaft tragen muss.

Die Daten sind sehr vollständig, da die Nachkontrollen in der Chemopräventionsstudie stringent kontrolliert werden. Die Intervallvorgabe geschah vor der Inklusion in die Studie und spiegelt somit "real life" wider. Fazit: Niedrigrisiko ist Niedrigrisiko. Wir müssen lernen, die richtigen Menschen zu spiegeln, also eine risikoadaptierte Vorsorge zu betreiben, die den Namen verdient und wie es auch das leider immer noch nicht umgesetzte Krebsfrüherkennungs- und Registergesetz vorsieht. Die Stiftung Lebensblicke hat sich mit diesem Thema ebenfalls schon in einem interdisziplinären Workshop auseinandergesetzt, vor dem Hintergrund, dass die moderne Technologie immer mehr kleine Polypen entdeckt, und auch vorgeschlagen, für Low-Risk-Adenome sogar bei fehlenden Risikofaktoren ein Intervall von bis zu zehn Jahren zuzulassen.

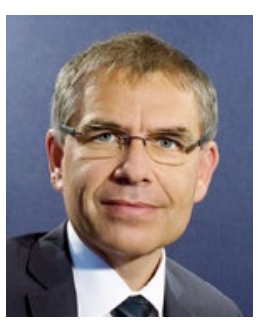

Prof. Dr. med. Dieter Schilling

Ärztlicher Direktor und Chefarzt der Medizinischen Klinik 2

Diakonissenkrankenhaus Mannheim Speyerer Str. 91 - 93, 68163 Mannheim E-Mail: Dieter.Schilling@diakonissen.de 